\title{
ABSOLUTE-VALUED ALGEBRAS
}

\section{KAZIMIERZ URBANIK ${ }^{1}$ AND FRED B. WRIGHT ${ }^{2}$}

An algebra $A$ over the real field $R$ is a vector space over $R$ which is closed with respect to a product $x y$ which is linear in both $x$ and $y$, and which satisfies the condition $\lambda(x y)=(\lambda x) y=x(\lambda y)$ for any $\lambda$ in $R$ and $x, y$ in $A$. The product is not necessarily associative. An element $e$ of the algebra $A$ is called a unit element if $e x=x e=x$ for any $x$ in $A$. Given any subset $B$ of $A, \operatorname{dim} B$ will denote the linear dimension of $B$; i.e., the power of a maximal set of linearly independent elements of $B$. Further, $[B]$ will denote the linear set spanned by the elements of $B$. For each $x$ in $A$, we shall denote by $A(x)$ the subalgebra generated by $x$. The algebra $A$ is called algebraic if $A(x)$ is finite dimensional for every $x$ in $A$. The algebra $A$ is said to be a division algebra if for every $a, b$ in $A$, with $a \neq 0$, the equations $a x=b$ and $y a=b$ are solvable in $A$.

An algebra over $R$ is called absolute-valued if it is a normed space under a multiplicative norm || ; i.e., a norm satisfying, in addition to the usual requirements, the condition $|x y|=|x| \cdot|y|$ for any $x, y$ in $A$. It is obvious that an absolute-valued algebra contains no divisors of zero.

A. A. Albert has shown [2, p. 768] that:

$\left(^{*}\right)$ An absolute-valued algebraic algebra with a unit element is isomorphic to either the real field $R$, the complex field $C$, the quaternion algebra $Q$, or the Cayley-Dickson algebra $D$.

F. B. Wright has proved $[6$, p. 332] the same theorem for absolute-valued division algebras with a unit element. In the present note we extend this result to an arbitrary absolute-valued algebra with a unit element.

First, we shall give a simple example of an infinite dimensional algebra which is absolute-valued. The existence of such an algebra shows that the assumption of the presence of a unit element is essential.

Let $A_{0}$ be the space of all sequences $x=\left\{x_{n}\right\}$ of real numbers with convergent series $\sum_{n=1}^{\infty} x_{n}^{2}$. $A_{0}$ is a Hilbert space over $R$ with respect to the norm $|x|=\left(\sum_{n=1}^{\infty} x_{n}^{2}\right)^{1 / 2}$, and with the usual addition and scalar multiplication: $\left\{x_{n}\right\}+\left\{y_{n}\right\}=\left\{x_{n}+y_{n}\right\}, \lambda\left\{x_{n}\right\}=\left\{\lambda x_{n}\right\}$. Let $\phi$ 1960.

Presented to the Society, January 29, 1960; received by the editors January 16 ,

1 Of the University of Wroclaw, Poland.

2 Research Fellow of the Alfred P. Sloan Foundation. 
be a one-to-one correspondence of the set of all pairs of natural numbers onto the set of all natural numbers. We define the multiplication of elements in $A_{0}$ as follows: $\left\{x_{n}\right\}\left\{y_{n}\right\}=\left\{z_{n}\right\}$, where $z_{\phi(m, k)}$ $=x_{m} y_{k}(m, k=1,2, \cdots)$. This product makes $A_{0}$ an algebra over $R$. Moreover, $A_{0}$ is absolute-valued. Indeed, we have the equality

$$
\begin{aligned}
|x y| & =\left(\sum_{n=1}^{\infty} z_{n}^{2}\right)^{1 / 2}=\left(\sum_{m=1}^{\infty} \sum_{k=1}^{\infty} z_{\phi(m, k)}^{2}\right)^{1 / 2} \\
& =\left(\sum_{m=1}^{\infty} \sum_{k=1}^{\infty} x_{m}^{2} y_{k}^{2}\right)^{1 / 2}=\left(\sum_{m=1}^{\infty} x_{m}^{2}\right)^{1 / 2}\left(\sum_{k=1}^{\infty} y_{k}^{2}\right)^{1 / 2} \\
& =|x||y| .
\end{aligned}
$$

Since $A_{0}$ is a Hilbert space, the function $|x|^{2}$ from $A_{0}$ to $R$ is a quadratic form admitting composition with respect to this multiplication. The complete structure theory of algebras (over any field) admitting such a form has been given by Kaplansky [4, p. 957], under the hypothesis that the algebra has a unit element. The algebra $A_{0}$ above shows that this hypothesis of existence of a unit element is essential to Kaplansky's results.

Throughout this paper, $A$ will denote an absolute-valued algebra.

Lemma 1. If all the elements of a subset $B$ of $A$ commute with each other, then $[B]$ is an inner-product space.

Proof. For every pair $x, y$ of elements of $B$ we have $(x+y)^{2}$ $-(x-y)^{2}=4 x y$. Consequently, for $|x|=|y|=1$, we get the inequality

$|x+y|^{2}+|x-y|^{2}=\left|(x+y)^{2}\right|+\left|(x-y)^{2}\right| \geqq 4|x| \cdot|y|$.

Hence, according to Schoenberg's theorem [5, p. 962], we know that $[B]$ is an inner-product space over $R$.

LEMMA 2. Let $x$ and $y$ be a pair of linearly independent elements of $A$. If $x$ commutes with $y$ and if $|x|=1$, then there exists an element $y_{0}$ such that $\left[x, y_{0}\right]=[x, y]$ and

$$
\left|\lambda x+\mu y_{0}\right|^{2}=\lambda^{2}+\mu^{2}
$$

for any $\lambda, \mu$ in $R$.

Proof. By Lemma 1, $[x, y]$ is an inner-product space. Since $x$ and $y$ are linearly independent, $[x, y]$ is a two-dimensional linear space. There is then an element $y_{0}$ with $\left|y_{0}\right|=1$ such that $y_{0}$ is orthogonal to $x$ and such that $[x, y]=\left[x, y_{0}\right]$. Equation (1) is a direct consequence of the orthogonality of $x$ and $y_{0}$. 
Lemma 3. Let $x, y \in A,|x|=|y|=1$, and $|x-y|=2$. If $x$ commutes with $y$, then $x+y=0$.

Proof. If $x=\lambda y, \lambda \in R$, we have the equalities $|\lambda|=|\lambda y|=|x|=1$, $|\lambda-1|=|\lambda y-y|=|x-y|=2$, which imply $\lambda=-1$. Thus $x+y=0$. Suppose then that $x$ and $y$ are linearly independent and commute with each other. Then, by Lemma 2 , there exists an element $y_{0}$ such that $[x, y]=\left[x, y_{0}\right]$ and

$$
\left|\lambda x+\mu y_{0}\right|^{2}=\lambda^{2}+\mu^{2} .
$$

The element $y$ can be written in the form

$$
y=\alpha x+\beta y_{0},
$$

where, by (2), we have

$$
1=|y|^{2}=\alpha^{2}+\beta^{2} .
$$

Furthermore, we have the equality

$$
4=|x-y|^{2}=\left|(1-\alpha) x+\beta y_{0}\right|^{2}=(1-\alpha)^{2}+\beta^{2} .
$$

From (4) we have $\alpha=-1$ and $\beta=0$. Substituting in (3) yields $x+y=0$.

TheOREM 1. An absolute-valued algebra with a unit element is isomorphic to either the real field $R$, the complex field $C$, the quaternion algebra $Q$, or the Cayley-Dickson algebra $D$.

Proof. By virtue of Albert's theorem (*), it is sufficient to show that every absolute-valued algebra $A$ with a unit element $e$ is algebraic. We will show that

$$
x^{2} \in[e, x]
$$

for any $x$ in $A$, which is equivalent to the inclusion $A(x) \subset[e, x]$, and which consequently implies the inequality $\operatorname{dim} A(x) \leqq 2$.

If $e$ and $x$ are linearly dependent, then (5) is obvious. Let us suppose that they are linearly independent. By Lemma 2, there exists an element $x_{0}$ such that

$$
[e, x]=\left[e, x_{0}\right]
$$

and such that

$$
\left|\lambda e+\mu x_{0}\right|^{2}=\lambda^{2}+\mu^{2} \quad(\lambda, \mu \in R) .
$$

From (6) follows the relation

$$
x^{2} \in\left[e, x_{0}, x_{0}^{2}\right] .
$$


Further, from (7) we have the equality

$$
\left|e-x_{0}^{2}\right|=\left|\left(e-x_{0}\right)\left(e+x_{0}\right)\right|=\left|e-x_{0}\right| \cdot\left|e+x_{0}\right|=2 .
$$

Since $e$ commutes with $x_{0}$ and since $|e|=1=\left|x_{0}\right|=\left|x_{0}^{2}\right|$, Lemma 3 asserts that $e+x_{0}^{2}=0$. Thus $\left[e, x_{0}, x_{0}^{2}\right]=\left[e, x_{0}\right]$. This, together with (6) and (8), gives relation (5). The theorem is thus proved.

The real field $R$ is the unique one-dimensional absolute-valued algebra. The structure of two-dimensional absolute-valued algebras is also well-known. In particular, every two-dimensional commutative absolute-valued algebra is isomorphic to either the complex field $C$ or to the algebra $C^{*}$ of all complex numbers with the usual addition and scalar multiplication, where the product of $x$ and $y$ is equal to $\bar{x} \bar{y}[1 ; 3]$.

TheOREM 2. If an absolute-valued algebra $A$ contains an element $a \neq 0$ which commutes with every element of $A$ and which is alternative, i.e., which satisfies the equations

$$
a(a x)=a^{2} x, \quad(x a) a=x a^{2},
$$

for any $x$ in $A$, then $A$ has a unit element.

Proof. We may suppose, without loss of generality, that $|a|=1$. If $a^{2}=\lambda a$, where $\lambda$ is in $R$, we may set $e=\lambda^{-1} a$, and we have

$$
\begin{aligned}
e^{2} & =\lambda^{-2} a^{2}=\lambda^{-1} a=e, \\
e(e x) & =\lambda^{-2} a(a x)=\lambda^{-2} a^{2} x=e^{2} x=e x .
\end{aligned}
$$

Hence we have the equation

$$
e(e x-x)=e(e x)-e x=0,
$$

which implies $e x-x=0$ for any $x$ in $A$. Since $e$ commutes with every element of $A, e$ is a unit element of $A$.

Now let us suppose that $a$ and $a^{2}$ are linearly independent. By Lemma 2, there exists an element $b$ such that

$$
\left[a, a^{2}\right]=[a, b]
$$

and such that $|\lambda a+\mu b|^{2}=\lambda^{2}+\mu^{2}$, for $\lambda, \mu$ in $R$. Hence we get

$$
\begin{aligned}
\left|a^{2}-b^{2}\right| & =|(a-b)(a+b)|=|a-b||a+b|=2, \\
\left|b^{2}\right| & =|b|^{2}=1 .
\end{aligned}
$$

Since $a$ commutes with every $x$ in $A$, we have, according to (9), $a^{2} x=x a^{2}$ for any $x$ in $A$. Therefore

$$
x y=y x \text { for } x \text { in } A, y \text { in }\left[a, a^{2}\right] .
$$


Taking into account (11), (12), and the relation $a^{2} b^{2}=b^{2} a^{2}$, we have, by virtue of Lemma $3, a^{2}+b^{2}=0$. From (10) we get the representation $b=\alpha a+\beta a^{2}$, where $\beta \neq 0$ since $a$ and $a^{2}$ are linearly independent. Hence $a^{2}+\alpha^{2} a^{2}+2 \alpha \beta a a^{2}+\beta^{2}\left(a^{2}\right)^{2}=a^{2}+b^{2}=0$. From (9) we have the equality

$$
a\left(\left(1+\alpha^{2}\right) a+2 \alpha \beta a^{2}+\beta^{2} a a^{2}\right)=a^{2}+b^{2}=0,
$$

which implies $\left(1+\alpha^{2}\right) a+2 \alpha \beta a^{2}+\beta^{2} a a^{2}=0$. Thus $a a^{2}$ is in $\left[a, a^{2}\right]$. Writing $a a^{2}=\lambda a+\mu a^{2}$, we have, according to (9),

$$
\left(a^{2}\right)^{2}=a\left(a a^{2}\right)=\lambda a^{2}+\mu a a^{2} \in\left[a, a^{2}\right] .
$$

Hence it follows that the product of an arbitrary pair of elements of $\left[a, a^{2}\right]$ also belongs to $\left[a, a^{2}\right]$. In other words, $\left[a, a^{2}\right]$ is a subalgebra of $A$, and $A(a)=\left[a, a^{2}\right]$. Hence $A(a)$ is a two-dimensional commutative absolute-valued algebra. Since the algebra $C^{*}$ does not contain an element $a \neq 0$ satisfying (9), $A(a)$ is isomorphic to the complex field $C$. Then there are elements $e_{0}, i_{0}$ such that $A(a)=\left[e_{0}, i_{0}\right]$, $e_{0}^{2}=e_{0}, i_{0}^{2}=-e_{0}, e_{0} i_{0}=i_{0} e_{0}=i_{0}$.

To prove the theorem, it is sufficient to show that $A(a)=A$. Let us suppose to the contrary, that there exists an element $x$ not in $A(a)$. Since, according to (13), $e_{0}$ and $i_{0}$ commute with $x$, then $\left[e_{0}, i_{0}, x\right]$ is an inner product space. There is then an element $y$ in $\left[e_{0}, i_{0}, x\right]$, with $|y|=1$, orthogonal to both $e_{0}$ and $i_{0}$. Consequently

$$
\begin{aligned}
& \left|y^{2}-e_{0}\right|=\left|\left(y-e_{0}\right)\left(y+e_{0}\right)\right|=\left|y-e_{0}\right|\left|y+e_{0}\right|=2, \\
& \left|y^{2}+e_{0}\right|=\left|\left(y-i_{0}\right)\left(y+i_{0}\right)\right|=\left|y-i_{0}\right|\left|y+i_{0}\right|=2 .
\end{aligned}
$$

By Lemma 3, $y^{2}-e_{0}=0$ and $y^{2}+e_{0}=0$, which implies the contradiction $e_{0}=0$. The theorem is thus proved.

TheOREM 3. A commutative absolute-valued algebra is isomorphic either to the real field $R$, or to the complex field $C$, or to the algebra $C^{*}$.

Proof. It is sufficient to show that every commutative absolutevalued algebra is at most two-dimensional. Suppose $\operatorname{dim} A \geqq 3$. By Lemma $1, A$ is an inner-product space. There exist orthonormal elements $i_{1}, i_{2}, i_{3}$ in $A$. From the equalities

$$
\begin{aligned}
& \left|i_{3}^{2}-i_{1}^{2}\right|=\left|\left(i_{3}-i_{1}\right)\left(i_{3}+i_{1}\right)\right|=\left|i_{3}-i_{1}\right|\left|i_{3}+i_{1}\right|=2, \\
& \left|i_{3}^{2}-i_{2}^{2}\right|=\left|\left(i_{3}-i_{2}\right)\left(i_{3}+i_{2}\right)\right|=\left|i_{3}-i_{2}\right|\left|i_{3}+i_{2}\right|=2,
\end{aligned}
$$

and from Lemma 3 , it follows that $i_{3}^{2}+i_{1}^{2}=0$ and $i_{3}^{2}+i_{2}^{2}=0$. Hence $i_{1}^{2}-i_{2}^{2}=\left(i_{1}+i_{2}\right)\left(i_{1}-i_{2}\right)=0$, which implies either $i_{1}+i_{2}=0$ or $i_{1}-i_{2}=0$. 
Since $i_{1}$ and $i_{2}$ are orthonormal, either is a contradiction. Hence $\operatorname{dim} A \leqq 2$.

\section{REFERENCES}

1. A. A. Albert, Absolute valued real algebras, Ann. of Math. vol. 48 (1947) pp. 495-501.

2. - Absolute valued algebraic algebras, Bull. Amer. Math. Soc. vol. 55 (1949) pp. 763-768.

3. - A note of correction, Bull. Amer. Math. Soc. vol. 55 (1949) p. 1131.

4. I. Kaplansky, Infinite-dimensional quadratic forms admitting composition, Proc. Amer. Math. Soc. vol. 4 (1953) pp. 956-960.

5. I. J. Schoenberg, $A$ remark on $M$. M. Day's characterization of inner-product spaces and a conjecture of $L$. M. Blumenthal, Proc. Amer. Math. Soc. vol. 3 (1952) pp. 961-964.

6. F. B. Wright, Absolute valued algebras, Proc. Nat. Acad. Sci. vol. 39 (1953) pp. $330-332$.

TUlane University OF Louisiana AND

University of Wroceaw, Poland 